Samain, Didier

\title{
Entropie et néguentropie de la traduction
}

Schweizerische Zeitschrift für Bildungswissenschaften 36 (2014) 2, S. 179-194

Quellenangabe/ Reference:

Samain, Didier: Entropie et néguentropie de la traduction - In: Schweizerische Zeitschrift für Bildungswissenschaften 36 (2014) 2, S. 179-194 - URN: urn:nbn:de:0111-pedocs-113613 - DOI: 10.25656/01:11361

https://nbn-resolving.org/urn:nbn:de:0111-pedocs-113613

https://doi.org/10.25656/01:11361

in Kooperation mit / in cooperation with:

\section{Schwe izerische Zeitschrift für Bildungswissenschaften \\ Rivista svizzera di scienze dell'educazione \\ Revue suisse des sciences de l'éducation}

http://www.rsse.ch/index.html

\section{Nutzungsbedingungen}

Gewährt wird ein nicht exklusives, nicht übertragbares, persönliches und beschränktes Recht auf Nutzung dieses Dokuments. Dieses Dokument ist ausschließlich für den persönlichen, nicht-kommerziellen Gebrauch bestimmt. Die Nutzung stellt keine Übertragung des Eigentumsrechts an diesem Dokument dar und gilt vorbehaltlich der folgenden Einschränkungen: Auf sämtlichen Kopien dieses Dokuments müssen alle Urheberrechtshinweise und sonstigen Hinweise auf gesetzlichen Schutz beibehalten werden. Sie dürfen dieses Dokument nicht in irgendeiner Weise abändern, noch dürfen Sie dieses Dokument für öffentliche oder kommerzielle Zwecke vervielfältigen, öffentlich ausstellen, aufführen, vertreiben oder anderweitig nutzen.

Mit der Verwendung dieses Dokuments erkennen Sie die Nutzungsbedingungen an.

\section{Terms of use}

We grant a non-exclusive, non-transferable, individual and limited right to using this document.

This document is solely intended for your personal, non-commercial use. Use of this document does not include any transfer of property rights and it is conditional to the following limitations: All of the copies of this documents must retain all copyright information and other information regarding legal protection. You are not allowed to alter this document in any way, to copy it for public or commercial purposes, to exhibit the document in public, to perform, distribute or otherwise use the document in public.

By using this particular document, you accept the above-stated conditions of use.

\section{Kontakt / Contact:}

\section{DeDOCS}

DIPF | Leibniz-Institut für Bildungsforschung und Bildungsinformation Informationszentrum (IZ) Bildung

E-Mail: pedocs@dipf.de

Internet: www.pedocs.de

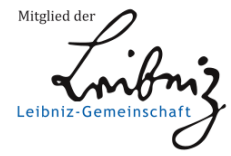




\section{Entropie et néguentropie de la traduction}

\section{Didier Samain}

En s'appuyant sur une expérience de traducteur et d'historien, l'auteur aborde la spécificité des concepts en sciences humaines et donc de leur traduction ou reformulation. À l'aide de métaconcepts descriptifs (troncation cognitive, effet de seuil), il montre qu'elle s'y accompagne tout à la fois de pertes et de gains d'information.

Sans nier l'intérêt des analyses culturalistes, il propose donc une approche plus historique et formelle, qui abandonne le topos de l'équivalence entre un original et sa copie pour lui substituer une conception métadiscursive des mécanismes de reformulation. L'analyse souligne simultanément l'ambiguïté de la notion de "traduction", illustrée entre autres par la notion quinienne de traduction radicale.

\section{Présentation}

\section{Quelques topoi}

Le nombre croissant de travaux qui leur sont consacrés témoigne de l'intérêt aujourd'hui suscité par les questions liées à la traduction au-delà du cercle des seuls spécialistes. Tout aussi révélatrice est la parution d'ouvrages de synthèse désormais bien documentés consacrés à l'histoire des perspectives traductologiques. Prunč (2007) fait par ailleurs apparaître une évolution: outre l'abandon explicite d'Übersetzung («traduction») au profit de Translation (moins usuel, et signifiant plutôt "transfert»), le sous-titre de l'ouvrage ${ }^{1}$ souligne d'emblée, non seulement le recul de la question des langues au profit de celle des cultures, mais aussi leur insertion récente dans des perspectives plus sociologiques, voire politiques. Cette réévaluation d'enjeux longtemps sous-estimés était souhaitable et nul ne saurait du reste nier que le simple choix de traduire un texte plutôt qu'un autre n'est jamais neutre, ni qu'il présuppose l'existence réciproque d'un horizon d'attente minimal. De manière plus générale, le tournant culturaliste aura contribué à renouveler la réflexion traductologique à un moment où les perspectives centrées sur les idiosyncrasies linguistiques et leurs hypothétiques corollaires cognitifs semblaient s'épuiser. La langue apparaît désormais 
comme une dimension, certes prégnante, mais une dimension seulement, des mécanismes de la reformulation.

Les lignes qui suivent n'adoptent toutefois, à proprement parler, ni une perspective culturaliste, ni une approche étroitement linguistique. Elles correspondent plutôt à la pratique d'un chercheur en sciences humaines, tout à la fois traducteur de textes culturellement situés et historien soucieux de modéliser autant que faire se peut les phénomènes qu'il observe. On se permettra à cet effet quelques rappels préliminaires.

Le fait d'abord que le traducteur se contente de démontrer la possibilité du mouvement en marchant. Et à l'aune des pas toujours singuliers, concrets, qu'il effectue, certaines topiques binaires usuelles en traductologie - entre familiarisation et Verfremdung, entre "sourcisme» et "ciblisme», entre perspective épistémique et perspective sociale, etc., paraissent inévitablement un peu générales. Quant à l'historien des sciences humaines, il lui est de même peu utile, pour les raisons que nous verrons, de se demander si la traduction entre dans un genre commun qui serait la reformulation, ou s'il faut la considérer comme le prototype de toute reformulation. De même, ni le traducteur, ni l'historien ne sont immédiatement concernés par cette trivialité qu'est la sous-détermination de la traduction ou cette curiosité qu'est son indétermination. La traduction radicale, ${ }^{2}$ qui choisit d'oublier que l'espace anthropologique, et donc langagier, n'est pas plus vide que l'espace physique, qu'il n'est donc jamais isomorphe et qu'il n'y a donc jamais indétermination, est éloignée de leur pratique effective. De l'hypothèse qu'aucune interprétation ne serait jamais certaine, il ne s'ensuit jamais que toutes les interprétations seraient également possibles. Mais nous reviendrons sur ce point.

L'écart entre thématiques convenues et pratique effective peut devenir criant. C'est le cas des clichés sur l'intraduisibilité et sur le relativisme langagier, que celui-ci se réclame d'un Sprachgeist dans ses versions traditionnelles, ou des «cultures» dans des versions plus récentes, comme si éviter le Charybde d'un universalisme naïf envoyait ipso facto l'interprète se cogner au Scylla du relativisme. Cette scolastique se décline sous de multiples formes, qu'on argue de la traductibilité effective pour justifier l'existence des universaux ${ }^{3}$, ou de l'intraductibilité pour les nier, ou encore du relativisme pour asserter l'intraductibilité, etc. Est-il besoin de rappeler cette banalité que si les interlocuteurs d'une même langue, ou de langues différentes, parviennent plus ou moins à s'entendre, c'est qu'en leur qualité de bipèdes sans plumes, ils habitent des univers tout à la fois divers et apparentés. Dans ses Grundfragen, P. Wegener (1885/1991) pose cette question volontairement banale: comment traduit-on et comprend-t-on Homère? Car si je dis en allemand (ou en français) qu'Ajax mange devant sa tente, je ne produis qu'une équivalence approchée. Les anciens Grecs en effet ne mangeaient pas comme nous, ni dans la même position corporelle. Et ce n'est pas en l'occurrence une question de langue! En d'autres termes, l'argument nous rappelle dans sa simplicité que, même au niveau le plus élémentaire qui 
soit, la langue ne fournit jamais autre chose que des indices (non des symboles!), des indices d'un certain rapport au monde. ${ }^{4}$ Et qu'il reste donc à faire la part des choses, lesquelles conduiront à une compréhension qui sera plus ou moins complète selon qu'elles sont plus ou moins partagées. ${ }^{5}$ Aux clichés précédents, il faut encore ajouter deux biais restés fréquents. Le premier repose sur une surévaluation du rôle de la langue. En soi la question linguistique en traduction est triviale - Qui songerait à nier l'existence de différences entre les systèmes linguistiques? - Mais ceci vaut à tous les degrés de l'échelonnement langagier, y compris entre les idiolectes. Le traducteur les gère tant bien que mal et les passe au besoin par pertes et profits. Et cela n'est pas spécifique, nous venons de le voir, à la traduction interlangue. Le malentendu, partiel, est constitutif de l'échange langagier ordinaire.

Le second cliché semble quant à lui profondément enraciné dans les représentations modernes de la traduction. Il s'agit de la vieille théorie de la mimésis, laquelle condamne la meilleure traduction à n'être jamais que la copie plus ou moins déformée d'un original. La prégnance de cette conception est telle qu'elle subsiste même chez les grands représentants de la tradition herméneutique. Ricœur (2004, p. 19) voit ainsi dans la traduction une «équivalence sans adéquation», comme si le "texte source» jouissait quant à lui d'une miraculeuse identité à soi, et il reprend du reste cette thématique en abordant le cas de la traduction intralangue. Auquel cas il s'agit, poursuit-il, de «dire la même chose autrement» (Ricœur, 2004, p. 45). «Nous retrouvons ainsi, à l'intérieur de notre communauté langagière, la même énigme du même, de la signification même, l'introuvable sens identique, censé rendre équivalente les deux versions du même propos».6

Or, outre qu'il est possible de concevoir les choses différemment, ainsi qu'on le verra tantôt, un apport majeur de la tradition herméneutique aura justement été de proposer une vision plus riche et positive de la reformulation, laquelle produit aussi de l'information, un supplément de sens par rapport au texte source. Toute traduction n'est donc pas condamnée à l'entropie. C'est là une thèse essentielle que l'historien des sciences humaines partage volontiers, avec cette différence qu'il ne voit pas dans cette reformulation l'actualisation d'une part implicite du sens légué par une tradition, comme le veut la tradition herméneutique. ${ }^{7}$ Il se contente de décrire la manière dont des concepts ou des systèmes sont repris, traduits et parfois servent à terme à autre chose. Il ne découvre donc ni réalisation d'un implicite, ni significations inaccomplies, mais, plus prosaïquement, des migrations de notions, entre les langues, entre les disciplines, ou simplement le long de l'axe temporel, ces différentes translations pouvant naturellement s'additionner. Ce sont ces mécanismes qu'on se propose d'exposer ici.

\section{Illustration}

En voici une première illustration. ${ }^{8} S^{\prime}$ il fut en France un livre de linguistique abondamment lu et commenté par les non linguistes, c'est bien le Cours de 
linguistique générale de Saussure (CLG). Les débats ultérieurs sur son caractère partiellement apocryphe ne changent rien au fait que ce texte, sous cette forme, a ainsi servi de matrice conceptuelle à plusieurs générations de chercheurs. Ce qu'on sait moins est que, loin d'être en rupture radicale avec un prétendu «positivisme» antérieur, il était en phase avec les problématiques de son époque, et notamment des maîtres de Saussure, c'est-à-dire les néogrammairiens de Leipzig. Sans doute la notoriété posthume du CLG a-t-elle en partie tenu à des facteurs externes - sa forme même, la langue utilisée et le lieu d'où il émanait.$-{ }^{9}$ Mais, sauf à supposer que ces facteurs fussent à eux seuls déterminants, qu'est-ce qui sépare le CLG et, par exemple, l'essai publié par Brugmann (1885/2009) peu auparavant, qui est tout aussi programmatique, et où apparaissent clairement les concepts de système, de synchronie, d'opposition, d'association? Une chose saute aux yeux à la lecture de ce texte: ces notions y sont intégrées dans deux réseaux qui ne sont plus directement visibles chez Saussure. Le premier est le débat sur les lois phonétiques qu'on peut sommairement schématiser ainsi: dans la perspective néogrammairienne, pour identifier une loi phonétique, il faut au préalable s'assurer qu'aucun effet de système (analogie ou autre) n'intervient dans l'apparition d'une forme donnée. Autrement dit, la perspective systémique (entendons synchronique) est essentielle chez Brugmann, mais elle est corrélée à une méthodologie, qui est celle de la grammaire historique. Le deuxième réseau est celui de la psychologie herbartienne, d'où viennent des termes comme Lautbild, «image acoustique», Bewegungsgefühl «sentiment kinesthésique», Vorstellungsgruppe "groupe notionnel» ou "groupe de représentations», qui sont usuels chez les néogrammairiens. Qu'observe-t-on par contraste chez Saussure? Quand ce dernier restitue implicitement Lautbild et Vorstellung par image acoustique et concept, ${ }^{10}$ ensuite glosés par signifiant et signifié, eux-mêmes empruntés aux logiciens médiévaux (significans/significatum), il détache un vocabulaire conceptuel du réseau argumentatif qui lui avait donné naissance et l'inscrit virtuellement dans un autre système. L'ancrage psychologique du vocabulaire néogrammairien cesse alors d'être visible. Il en va de même de la synchronie saussurienne, qui n'exhibe pas davantage son fondement diachronique.

L'effacement des fondements heuristiques des concepts utilisés produit ainsi un effet de seuil, qui permettra par exemple à des épigones d'assimiler synchronie et achronie, ce qui eût été un non sens pour Brugmann et l'eût été tout autant pour Saussure. À cela près qu'une lecture «amnésique» du CLG est techniquement possible, ce qui n'est pas le cas du livre de Brugmann. On peut formuler l'hypothèse qu'il s'agit d'un phénomène assez général, ${ }^{11}$ et considérer que cette troncation cognitive est probablement nécessaire pour qu'un texte en sciences humaines fasse office de matrice conceptuelle. 


\section{Les tropismes systémiques}

\section{Termes, notions, systèmes}

Cette migration des notions d'un espace disciplinaire et/ou linguistique à un autre constitue la matière première de l'historien des sciences et l'exemple précédent suggère qu'elle ne s'opère pas au hasard, mais plutôt selon un ordre de démotivation et de terminologisation croissante. En voici un exemple simple, qui a été analysé ailleurs $^{12}$ et qui montrera au passage que ce phénomène touche les deux faces du signe. Il s'agit de l'emploi du mot Stellenwert par le linguiste slavisant E. Koschmieder (1929/1971). Issue du vocabulaire ordinaire, cette notion avait été introduite peu auparavant par le psychologue R. Hoenigswald (1920) pour distinguer du temps physique le temps vécu (erlebte Zeit), dans lequel les événements reçoivent pour le Moi une Stellenwert in der Zeit, une position dans le temps. Cette dimension phénoménologique se retrouve chez Koschmieder mais prise comme un simple donné (elle devient même un terme: Zeitstellenwert), et surtout, elle assure chez lui une fonction technique, absente chez le psychologue, puisqu'elle joue un rôle central dans sa théorie de l'aspect verbal. Ce double phénomène de condensation lexicale et d'intégration technique, qui fait que la copie se révèle finalement plus compacte que son modèle, est un effet de seuil analogue, même si ses conséquences furent bien plus discrètes, aux phénomènes précédemment observés.

Ce mécanisme de troncation, qui joue donc un rôle important dans la transmission des concepts, pourrait toutefois donner à penser que ces reformulations s'accompagnent d'une perte d'information conduisant à terme à des changements d'état (e.g. le passage de la phénoménologie à la linguistique). S'agissant du matériel argumentaire, cette perte est indéniable puisqu'elle est constitutive du processus lui-même. Mais une conception purement entropique (la «copie» comme image appauvrie d'un «modèle») occulterait la complexité des mécanismes qui entrent en jeu ici, car la reformulation se traduit simultanément par des gains de signification. Cette remarque rejoint à sa manière l'intuition herméneutique. C'est ce que nous allons voir dans la suite de ce travail. Nous reviendrons in fine sur le changement d'état.

Mais il nous faut d'abord mentionner deux caractéristiques, du reste liées, des concepts utilisés en sciences humaines. Le fait d'abord que, du point de vue logique, ce sont souvent des objets en quelque sorte intermédiaires: ils sont en principe suffisamment explicites et thématisés pour être de vrais outils d'analyse, ce ne sont plus des notions du langage ordinaire. Néanmoins, ce ne sont pas non plus stricto sensu des termes scientifiques, en ce sens qu'ils ne sont pas toujours constructibles par une méthode. Leur deuxième trait majeur est leur historicité. Un livre de grammaire par exemple, n'exprime pas une théorie grammaticale, il est le fruit d'une sédimentation de modèles hétérogènes où le spécialiste identifiera des éléments issus de la tradition latine, de la théorie port-royaliste de la proposition, plus récemment des approches pragmatiques, etc. Ce n'est qu'a 
posteriori, qu'on observe des tentatives d'homogénéisation. En linguistique, les grammaires valencielles sont ainsi un bel exemple d'articulation entre morphologie et syntaxe, mais cette articulation n'est justement jamais donnée. L'histoire transmet bien des unités fonctionnelles (les concepts grammaticaux), mais non un système intégré dans lequel elles s'inscriraient. Corrélativement à cette hétérogénéité factuelle, on observe donc une sorte de tropisme vers la systématicité qui conduit dans quelques cas privilégiés (ainsi dans le CLG) à un quasi équilibre terminologique.

Mais alors, si un ouvrage de sciences humaines n'est au mieux que partiellement homogène, s'il n'est pas uniquement composé de concepts méthodologiquement construits et organisés en système, mais plus souvent de notions diverses, quelles en sont les conséquences pour le traducteur? Il arrive à tout lecteur de se demander: que veut dire Un Tel, en employant tel mot? Et de ne pouvoir y répondre. Le problème est que le traducteur, lui, n'a pas le choix, il lui faut savoir s'il a ou non affaire à un terme, à défaut s'il s'agit du moins d'un concept en attente de terminologie. Ou de notions qu'il suppose en voie de conceptualisation. Que lui suppose telles, plus rarement l'auteur lui-même.

\section{Les mots hors réseau}

Les limites de la systématique en sciences humaines se révèlent avec une évidence particulière lorsqu'on voit réapparaître le langage ordinaire au sein même du discours académique. Songeons, pour nous borner à des exemples connus, à ces mots si galvaudés en sociologie que sont misère (Bourdieu), ou peuple (Rancière, Michéa ou Lasch aux USA) ${ }^{13}$. Certes, ce ne sont pas n'importe quels mots, ni n'importe quels auteurs. Mais ceci n'explique pas pourquoi des notions évacuées, non sans raison, du lexique des sciences humaines peuvent ainsi réapparaître, comme si leurs utilisateurs s'efforçaient par là d'enfoncer les doigts dans la chair du réel ou à tout le moins de saisir quelque chose de ce réel que le maillage de la langue technique aurait laissé échapper. Il est permis de regretter ce retour aux notions ordinaires, et même de considérer qu'un tel geste est largement fantasmatique. Reste ce fait que, pour recharger ou renouveler la connaissance des choses dont s'occupent les sciences humaines, il n'est pas toujours fait appel à un surcroît d'analyse, mais parfois aussi au langage ordinaire. Il serait ridicule de faire de ce constat une généralité. Le mouvement inverse, de terminologisation, est évidemment constitutif de toute science. Comparons cependant cela à des mots bien balisés du même champ idéologique comme prolétaire ou infrastructure. $S$ 'il est facile de donner à entendre, rationnellement, ce que désignent des mots, c'est qu'ils sont des nœuds dans un réseau, au point que, pour les invalider, le moyen le plus radical reste d'en invalider le code, le système, dans lequel ils prennent sens. Pareille stratégie n'est tout simplement pas possible avec peuple et misère, puisque ces notions qu'on ne peut qu'expériencer, fût-ce indirectement, et non construire, opposent une limite de principe à toute approche sémiotique de l'interprétation. Elles sont hors réseau et passent donc entre les mailles du 
filet terminologique. D'où l'embarras qu'elles suscitent chez les sociologues et, corrélativement, l'obstacle qu'elles opposent à la traduction. Ce n'est là, à tout prendre, qu'une expérience banale. Sauf qu'il ne s'agit pas ici de littérature ou de vécus anthropologiques, mais bel et bien de sciences. Le paradoxe peut être ainsi formulé: une partie du vocabulaire des sciences humaines n'est pas composé de symboles dans l'acception stricte du terme, ce qui peut en hypothéquer la transmissibilité. Rappelons-nous l'apologue d'Ajax devant sa tente.

Est-ce une raison suffisante pour les renvoyer à la spécificité d'une culture, qui incarnerait un «autre» réseau, pourvu de ses propres nœuds? Non, bien sûr. Même si on peut y voir des analogies avec la question des cultures, il s'agit là d'un problème plus général, d'ordre logique. Au reste, on peut élargir au passage le propos. Les thèses d'un certain structuralisme franco-français ont fait long feu, et plus personne ne songe à présenter les cultures (ou les textes, ou tout ce qu'on voudra) comme des réseaux bien construits. Les observations précédentes suggèrent au contraire qu'une partie de la production d'une culture échappe nécessairement au code de cette culture même. Et il ne s'agit nullement d'implicite, ou de refoulé, ou d'une quelconque figure de l'autre. Il s'agit de ce fait sémiotique qu'on ne peut pas se passer de codes, que ces codes sont composés de points remarquables (pensons aux points remarquables en géométrie analytique ou aux phénomènes de prégnance dans la gestalt), et que ce qui ne correspond pas à ces points remarquables, soit n'existe pas - c'est tout simplement dépourvu de sens -, soit fait l'objet d'une correction par équivalence approchée. Il existe de bonnes formes discursives comme il est de bonnes formes perceptives. Sous la pression de ces bonnes formes discursives, il n'est du reste par rare qu'une traduction rectifie, parfois brutalement, un original «hors réseau». Nous allons le voir dans un instant.

\section{Illustration}

Le traducteur est contraint de prendre acte de cette dialectique inhérente au mode de conceptualisation des sciences humaines, tendu entre ces deux pôles que sont, d'un côté les sédimentations toujours aléatoires de l'histoire, de l'autre le tropisme vers le système, d'un côté le maillage du réseau terminologique, de l'autre les notions surgies entre ces mailles. S'il arrive que les reformulations conduisent à des effets de seuil, ainsi qu'on l'a vu en évoquant la ligne Herbart-néogrammairiens-Saussure, ces textes seuils apparaissent minoritaires et occupent de ce fait une position privilégiée. Ils jouent le rôle de «bonnes formes» par rapport auxquelles il est tentant de situer les autres textes.

L'œuvre de Gardiner et sa traduction française en fournissent un exemple d'école. ${ }^{14}$ Cet égyptologue, linguiste amateur, a publié en 1932, sous le titre Theory of Speech and Language, un livre qui héritait de plusieurs réseaux conceptuels hétérogènes, allant de la grammaire générale à la pragmatique en passant par des restes de psychologie allemande. Cette diversité apparaît dans le vocabulaire employé. Ainsi, s'agissant de language et speech, à côté de pôles d'attraction termi- 
nologique, correspondant peu ou prou au couple post-saussurien «langue»vs. "discours", on relève des emplois non intégrés relevant, soit du langage ordinaire, soit de modèles épistémologiques antérieurs. L'ouvrage a bénéficié d'un regain d'intérêt à partir des années 70 , lorsqu'on y a vu une préfiguration de la pragmatique, ce qui lui a valu d'être traduit en français (Gardiner, 1989). Or, indépendamment du caractère conjoncturel du titre choisi pour l'édition française, ${ }^{15}$ ce qui frappe le lecteur de cette traduction n'est pas tant la volonté, somme toute compréhensible, de contextualiser ce texte par rapport à une modernité supposée, qu'un embarras terminologique à peu près constant, y compris avec ce qui fait pourtant l'épine dorsale de l'ouvrage, à savoir speech vs. language. Speech est ainsi rendu, tantôt par «discours», tantôt par «langage», tantôt par «langue»; language l'est tantôt par «langage», tantôt par "langue», tantôt par «langue maternelle». À quoi il faut ajouter des effets de phraséologie, ainsi lorsque act of speech devient acte de langage, induisant une fausse reconnaissance avec Austin. (L'expression visait en réalité à fournir un équivalent de l'allemand Äusserung, qui désigne à l'époque une occurrence singulière de parole, sans grand rapport donc avec les speech acts d'Austin.) De même, origin of speech, qui désigne bien chez Gardiner l'origine de la parole, est traduit par origine du langage, ce qui fait tout autant contre-sens. Les biais notionnels et phraséologiques sont ici patents.

Que s'est-il passé finalement? Ne chicanons pas, il s'agit d'une traduction contestable d'un ouvrage qu'on hésitera à qualifier de majeur. Cela étant, l'échec lui-même est intéressant, puisque l'impératif de traduire met donc mécaniquement au jour une caractéristique du texte original, à savoir l'hétérogénéité de son lexique conceptuel. Ajoutons que les biais cognitifs induits par l'horizon d'attente sont eux-mêmes des faits historiques, à considérer comme tels, qui induisent une rectification quasi gestaltiste d'un texte qui ne présente pas, et pour cause, une bonne forme. L'idiome de réception n'a pas oblitéré la transparence supposée du message initial, il a augmenté la cohérence d'un ensemble qui en est largement dépourvu. ${ }^{16}$

\section{Néguentropie}

\section{Le travail du traducteur}

Le degré de conceptualisation et a fortiori de terminologisation d'un vocabulaire est en revanche rarement évident à la lecture cursive. Les mots et expressions auxquels a affaire le traducteur ne sont au départ que des indices dont il cherche à définir le sens par récurrence (en collationnant des occurrences), puisqu'il ne peut se résigner à cette incertitude inhérente à la lecture ordinaire. Ce repérage sémantique, qui a pour but de faire apparaître des tropismes systémiques qui ne sont pas directement visibles, procède donc d'une stratégie différente d'une situation de compréhension immédiate, ou du réglage pragmatique d'une signification en discours, car une ouvre entière n'est pas un "contexte» dans l'acception linguistique du terme. 
Autrement dit, le résultat obtenu est bel et bien le fruit d'une opération métadiscursive, voire métalinguistique, qui organise le matériel de départ. Les notions souvent peu explicites qu'on trouve dans la Sprachtheorie de Bühler $(1934 / 2009)^{17}$ en fournissent de nombreuses illustrations. En allemand, il y a par exemple deux termes à la date pour désigner les dénominatifs: Nennwort, litt. «mot de nomination», et Begriffszeichen, litt. «signe de concept». Le traducteur n'a aucun moyen, ni le lecteur germanophone, de savoir d'office si ces deux mots sont ou non chez Bühler des variantes libres pour désigner ce qu'on appelle en français des dénominatifs. Seul un relevé des collocations a pu montrer que, dans la Sprachtheorie, Nennwort entre corrélation systématique avec Zeigwort, "déictique», alors que Begriffszeichen relève d'une sémiotique générale, et désigne tout aussi bien le catégorème (par opposition au syncatégorème) que le nom commun (par opposition au nom propre), etc. Or non seulement cela ne se révèle, il faut y insister, qu'à la suite d'une opération particulière, mais c'est donc seulement dans la traduction française que ce distinguo apparait clairement, puisqu'il a bien fallu réserver le terme technique dénominatif au seul Nennwort, en traduisant donc Begriffszeichen, quand c'était nécessaire, d'une périphrase: «signe linguistique de concept» et, quand on pouvait s'en dispenser, plus simplement par «nom».

D'autres cas sont bien plus complexes que cet exemple simple, qui suffira cependant à montrer comment le traducteur est ainsi amené, mécaniquement encore, à produire un surcroît de terminologie. Même quand la traduction s'efforce d'être fidèle à l'esprit du texte traduit et d'éviter les rectifications du type observé précédemment, de tels effets de sursémantisation apparaissent difficilement évitables. Nous avons vu comment Saussure disjoint le couple Lautbild/ Vorstellung de son enracinement psychologique et l'insère dans un réseau différent, plus sémiotique. Mais quand les psychologues ou les philosophes font l'inverse, qu'ils choisissent de traduire Vorstellung par "représentation», ils ne sont guère plus fidèles au sens originel. Ils lui sont infidèles différemment, par sursémantisation, car une Vorstellung, en soi, c'est tout simplement une idée au sens de la grammaire générale. - Les Vorstellungen d'Un Tel sur tel problème, ce ne sont pas ses représentations mentales, ce sont juste ses idées sur la question! Mais l'histoire piège ici les traducteurs. Les psychologues allemands ont en effet donné une épaisseur empirique à ce mot du langage ordinaire, avec des questions qui étaient au demeurant tout à fait pertinentes. Par exemple, est-ce que toutes les opérations mentales sont des Vorstellungen? Est-ce que toute Vorstellung est obligatoirement corrélée à une Anschauung, une intuition sensible? Il s'agissait de vraies questions scientifiques, dont la dernière n'a reçu de réponse, négative, qu'au début du $20^{\mathrm{e}}$ siècle. Mais le résultat, depuis que ces questions se sont sédimentées sur le mot Vorstellung, est qu'il n'est plus guère possible, en contexte technique, de le traduire en français par idée comme il faudrait, idéalement, le faire. Le traducteur est ainsi condamné à dire représentation. Le problème est que représentation est un terme. Vorstellung n'est pas un terme. 


\section{Le supplément dictionnairique}

Les exemples précédents montrent comment la traduction, et plus généralement, la reformulation, entrainent tout à la fois perte et accroissement d'information. Toutefois nous avons pour le moment implicitement admis que, nonobstant ces différences de cohésion, le "texte source» et le «texte cible», 18 appartiennent au même univers logique. À tout prendre, c'est un peu inexact. La manière dont le traducteur procède, par collations d'occurrences et de contextes, en intervenant, volontairement ou non sur les irrégularités du texte source, tout cela, tend déjà, ainsi qu'on l'a souligné, à faire de son travail un peu plus qu'une version améliorée d'un modèle. La "copie» s'apparente autant à un métadiscours qu'à une paraphrase.

Mais nous allons voir maintenant que l'idée même de traduction véhicule occasionnellement un implicite métalinguistique bien plus prononcé. La difficulté tient en fait à la polysémie du mot "traduction» qui regroupe des pratiques qui ne different pas seulement par leurs objets empiriques (e.g. des textes «techniques» vs. des textes littéraires ou de sciences humaines) ou par leurs modalités concrètes (e.g. la traduction simultanée vs. la traduction d'un texte écrit), mais bien par leur nature. Pour comprendre ce dont il est ici question, le dictionnaire peut servir de référentiel. - Qu'est-ce qu'un dictionnaire? Ce n'est pas simplement un stock de mots pourvus d'équivalents dans la même langue ou dans une autre. Bien plus encore qu'une traduction, un dictionnaire est une machine à produire de l'entropie négative. Par sa simple existence, la nomenclature alphabétique produit en effet une cohérence minimale, elle lisse ses objets transformés en unités lexicales. Le dictionnaire révèle des apparentements lexicaux, des systèmes dérivationnels, etc. Il ne se contente donc jamais d'indiquer le sens des mots, il est un facteur essentiel de structuration du lexique, qu'il contribue à créer et à organiser. Dans le dictionnaire, un ordo nominum, ou plus exactement ordo partium orationis (soit des unités du lexique régies par des relations formelles), remplace la connexio rerum qui caractérise les mots du monde ordinaire. Or cette différence, évidente, de statut logique entre les mots employés ordinairement - dans la vie normale, les mots, on les emploie, on ne les définit pas - et les unités lexicales du dictionnaire, cette différence semble parfois ignorée lorsqu'on parle de traduction.

La thèse quinienne sur l'indétermination de la traduction en fournit une illustration intéressante. Imaginons, dit Quine (1960) dans l'un des passages les plus célèbres de Word and Object, qu'un linguiste en contact avec une langue radicalement inconnue entende un informateur prononcer gavagai au passage d'un lapin. La répétition d'expériences de ce genre lui permettra sans doute de corréler ce type d'énoncé à des situations, mais en aucun cas de savoir si gavagai signifie effectivement «lapin» et non, plutôt, «il y a un lapin», ou encore «segment de lapin», voire «lapinité».

Une objection volontairement naïve à cet argument serait de demander: qu'est-ce qui ressemble plus à un chasseur qu'un autre chasseur? Et, sur cette 
base, d'arguer qu'en Europe ou en Amérique, sinon dans cette jungle putative, les attributions de quiddité lapine sont rarement la tasse de thé, ou pinard, des intéressés, et que, lorsqu'ils déchargent leurs chevrotines, ce n'est pas davantage sur des segments de lapin. Ce serait la réponse du technicien au mathématicien tenté d'oublier que la traduction radicale repose sur la même fiction méthodologique que la mécanique classique, l'existence d'un espace vide, uniforme, dans lequel tous les événements seraient en soi équiprobables. La commodité ou le rendement de cette fiction ne changent rien au fait qu'il s'agit d'une simple construction intellectuelle. Rien de tel n'existe dans le monde réel, physique ou anthropologique, dans lequel il est certes possible à un philosophe ou à un grammairien de se pencher sur la lapinité ou la lapinitude. Quant aux chasseurs, c'est bel et bien aux lapins qu'ils s'intéressent en priorité. ${ }^{19}$

Naturellement cette objection n'atteint pas le cœur de l'argument quinien, qui, délesté de son apologue exotique, revient à dire qu'un énoncé (utterance) n'indique pas par lui-même sa composition; qu'il n'est donc accessible qu'à une approche strictement behavioriste (tel énoncé est corrélé à tel comportement ou situation), tandis que sa sémantique interne reste, sinon en fait du moins en droit, inaccessible à l'expérimentation. Admettons donc cette fiction d'un espace langagier isomorphe. Alors, effectivement, l'argument est imparable. Plus exactement, il l'est pour autant que nous restions enfermés dans l'alternative à laquelle cet apologue nous conduit: soit rejeter cette fable pour des raisons anthropologiques, et il est alors impossible d'en discuter l'argument, soit l'accepter à titre de fiction méthodologique, et il est alors impossible de le réfuter.

Mais quid de cette alternative elle-même et de la conception de la traduction qu'elle révèle? On peut en effet reformuler différemment la question anthropologique: qui, sauf un grammairien ou un lexicographe, peut avoir l'idée de passer outre l'anisotropie effective de l'espace langagier et de faire comme si nous avions juste affaire à des mots (tous égaux en droit) et à des structures? C'est même une idée de grammairien tardif, au vu du temps qu'il aura fallu pour étendre le concept de partie du discours à tout type d'occurrence lexicale. Elle ne viendrait certes pas à l'esprit d'un chasseur. Viendrait-elle à l'esprit d'un traducteur? Notre linguiste imaginaire piste à la trace les unités lexicales et les significations. Mais qui, à par lui et les gens qui lui ressemblent, cherche de tels objets, et surtout de cette façon? Les mots du monde ordinaire, disions-nous, on les emploie, on ne les chasse, ni ne les classe.

Autrement dit, derrière la fiction de la nature isotropique de l'espace langagier, se cache un présupposé structurant de la réflexion grammaticale, en l'occurrence ce privilège accordé à la nomination et l'identification des unités dans la détermination du sens. Or il s'agit là, Quine lui-même en conviendrait sans doute, d'un artefact sémiotique. Et ni cet artefact, ni son corollaire, la signification, ne sont en soi inévitables, comme le montre du reste, parmi les théories récentes du langage, l'approche énactive et behavioriste d'un Maturana, ${ }^{20}$ qui évacue purement et simplement la représentation. Ou, pour dire les choses autrement 
encore, l'argument quinien ne fait sens que sur le fond du postulat sémiotique constitutif du métalangage grammatical. Ce postulat n'est pas illégitime, plus exactement, il n'est ni plus ni moins légitime que la grammaire occidentale. Et cela signifie, tout simplement, que ce que Quine présente comme «traduction radicale» est une situation de métalangage, un artefact dictionnairique, et non un problème d'équivalence entre un énoncé inconnu, gavagai, et son hypothétique traduction.

\section{Dolmetscher vs. Übersetzer.}

L'auteur consacre des pages, pertinentes, à la différence - autant dire l'incommensurabilité logique - entre, d'une part, un énoncé Gavagai ou Lapin (il faut ici comprendre par énoncé une séquence concrète observable), passible d'une approche qu'on sera tenté d'appeler aujourd'hui énactive, en tout cas behavioriste, et, d'autre part, ce qu'il appelle (dans une acception différente de celle utilisée ici) les termes, c'est-à-dire les mots ou séquences de mots gavagai ou lapin. ${ }^{21} \mathrm{Si}$ on la résume, la thèse de l'indétermination de la traduction consiste donc à montrer que le passage d'un plan à l'autre est impossible à partir des seules ressources de la langue, puisqu'aucun énoncé ne peut par lui-même indiquer sa structure. C'est là un point philosophiquement important que Quine dégage avec une grande clarté. Quoi qu'il en soit, traduire, tel que le conçoit Quine, impliquerait précisément de passer de l'énoncé à la structure, c'est-à-dire un changement d'état logique. On a ici le sentiment que l'auteur, en demandant si la signification d'une entité est radicalement accessible, et en y répondant bien évidemment par la négative, est comme retenu par un infime reste de physicalisme. La conséquence est pourtant simple: Rabbit-sentence et rabbit-word ne sont pas séparés par des «niveaux d'organisation» au sein d'une même trame empirique, il y a entre eux le hiatus qui sépare un langage d'un métalangage.

L'allemand moderne dispose, comme on sait, de deux mots pour désigner le traducteur, Dolmetscher et Übersetzer. Le Dolmetscher, c'est le «truchement» du français classique, celui qui fera par exemple aujourd'hui de la traduction simultanée. L'Übersetzer traduit quant à lui des textes, à coup de dictionnaires et de collations d'occurrences. Bon gré mal gré, l'Übersetzer est peu ou prou linguiste. Il produit, bon gré mal gré, du métalangage, ainsi que nous l'avons vu plus haut. Le Dolmetscher fait tout autre chose, il fait comme les locuteurs ordinaires, il emploie les mots. Le défaut de l'argument quinien est sans doute d'avoir imaginé un Dolmetscher se comportant comme un linguiste. 


\section{En guise de conclusion}

Ce qui n'est qu'esquissé chez Quine, c'est donc ce saut qualitatif, le changement d'ordre qu'implique le passage de l'énoncé à la structure, et qui est l'opération constitutive d'une description grammaticale. Il ne viendrait donc pas à l'esprit de l'auteur de ces lignes de demander si une signification est ou non «accessible». Il va de soi qu'elle n'est pas «accessible», puisqu'elle n'est pas d'ordre empirique! L'énoncé d'une part, dans sa dimension énactive, et la signification d'autre part, un biologiste comme Maturana le dit à manière, sont des incommensurables logiques, pour cette raison simple - faut-il le répéter? - que structure et parties du discours ne sont pas des réels, mais des produits du métalangage, des suppléments d'information. Analyser un énoncé, ce n'est donc ni le traduire, ni le comprendre, c'est lui faire subir un changement d'état logique.

Dans ces conditions, la conclusion réciproque s'impose: c'est une faute logique que de présenter la traduction (entendons ce que fait l'Übersetzer) comme une translation, c'est-à-dire selon la métaphore d'un déplacement au sein d'un espace. Ce qu'on appelle en jargon le «texte source» et le «texte cible» étant censés entrer en relation d' "équivalence sans adéquation» (Ricœur). Cette conception est indéfendable, et sans doute même à des niveaux très élémentaires: si je dis le sens de ce qu'a dit mon voisin, je ne dis pas ce qu'il a dit, mais autre chose et bien davantage. Les pages qui précèdent se sont au contraire proposé de montrer comment la reformulation crée de la structure, augmente le différentiel d'information. Il serait donc souhaitable, si l'on cherche à formaliser un tant soit peu les mécanismes de traduction et de reformulation, de remplacer le topos de l'équivalence par le modèle du métalangage.

\section{Notes}

1 Von den Asymetrien der Sprachen zu den Asymetrien der Macht.

2 Quine (1960) appelle radical translation une situation dans laquelle un linguiste se trouverait s'il lui fallait interpréter des énoncés d'une langue totalement inconnue en se fondant uniquement sur les contextes dans lesquels ils sont employés. On reviendra in fine sur cette expérience de pensée qui sert d'argument à la thèse quinienne de l'indétermination de la traduction.

3 Rastier (1995) rappelle que c'est en effet là l'un des arguments traditionnels du rationalisme grammatical. Mais il n'est pas indifférent que celui-ci se retrouve sous une forme, certes plus nuancée, même chez Hagège par exemple (1986, pp. 9-10)

4 Une perspective empiriste cohérente conduit logiquement, soit à rejeter l'idée même de signifié (et donc de "symbole»), au motif que toute signification s'enracine dans l'expérience individuelle et que les signes ne sont que les traces mémorisées de ces expériences, soit à l'accepter au titre d'artefact de la description (mais en aucun cas comme propriété effective des signes). La position de Wegener est ici drastique: Ce n'est en effet, selon lui, que la forme "mécanisée» de la compréhension de choses qui crée l'illusion de puiser le sens de l'énoncé dans les mots et les formes grammaticales. (1885, pp. 114-115.)

5 Sur le problème des «choses», Samain (2011).

6 Ricœur, 2004. Ajoutons que ce modèle synonymique conduit de surcroît, ainsi que Ricœur le souligne lui-même en évoquant l'argument quinien, aux apories du troisième 
homme: pour évaluer l'équivalence de $\mathrm{P}_{1}$ et de $\mathrm{P}_{2}$, il faudrait disposer d'un énoncé $\mathrm{P}_{3}$, et ainsi de suite.

7 Par exemple dans Buck (1976).

8 Je ne puis reprendre le détail de cette illustration, exposée dans Samain (2014).

9 Genève et Prague étaient, avant leur essor, des lieux périphériques.

10 Par une sorte de restitution inverse, le traducteur allemand du CLG a naturellement rendu concept par Vorstellung et non, bien sûr, par Begriff.

$11 \mathrm{Ni}$ le changement d'auteurs, ni le changement de langue ne sont en soi nécessaires. Fortis (à paraître) en fournit une illustration dans un travail consacré à l'héritage néogrammairien de Bloomfield. Il montre en effet qu'il n'y a pas eu "deux» Bloomfield, l'un héritant de Herbart et l'autre se réclamant de Watson, mais que les reformulations successives des mêmes propositions les ont progressivement décantées de leur contenu substantiel (en l'occurrence l'appareil explicatif herbartien), jusqu'au moment où elles n'ont plus été que des énoncés techniques. Il en a résulté un effet de seuil à partir duquel l'antimentalisme propre au behaviorisme américain a pu se développer.

12 Koschmieder, 1996, pp. xx-xxv.

13 Bourdieu, P. (1993). La misère du monde. Paris: Seuil. Lasch, C. (1995). The Revolt of the Elites: And the Betrayal of Democracy. New York: W.W. Norton \& Co. Michéa, J.-C. (1998). Les intellectuels, le peuple, et le ballon rond. Montpellier: Climats.Rancière, J. (1990). Court voyage au pays du peuple. Paris: Seuil. Rancière, J. (2003). Les scènes du peuple. Lyon: Horlieux.

14 Pour le détail, Candel \& Samain, 2013.

15 Langage et acte de langage. Aux sources de la pragmatique.

16 On propose ici une version assouplie du discontinuisme kuhnien, en avançant la thèse qu'il existe dans l'histoire des idées des positions d'équilibre conceptuel et terminologique, par rapport auxquelles les autres notions tendent à se définir, car il s'agit aussi de forme. La notion d' «idiome de détresse» peut fournir ici une analogie commode: du point de vue ethnologique, l'important n'est pas le constat banal qu'existent des «traductions» de la détresse, mais que l'idiome en question offre une structure d'accueil, culturellement héritée. (Nichter, 1981).

17 Bühler, 1989, pp. 617-668.

18 Ces termes sont utilisés comme de simples étiquettes, sans tenir compte de leurs présupposés théoriques.

19 Lia Formigari (2007, pp. 114-118) avance du reste des objections voisines tant à Chomsky qu'à Quine.

20 Par exemple Maturana,1987; Maturana, Mpodozis \& Letelier, 1995.

21 Quine, 1960, pp. 68-79. Quine use d'un artifice typographique pour distinguer Rabbit (sentence) et rabbit (word).

\section{Références}

Brugmann, K. (2009[1885]). Zum heutigen Stand der Sprachwissenschaft. Cambridge: Cambridge University Press.

Buck, G. (1976). La structure de l'expérience herméneutique et le problème de la tradition. Social Science Information, 15, 239-267.

Bühler, K. (1989). Théorie du langage. Préface par Jacques Bouveresse, présentation par Janette Friedrich, traduction, notes et glossaire par Didier Samain. Marseille: Agone.

Candel, D. \& Samain, D. (2013, sous presse). Translation as a Historically Situated Activity. Situational Issues: the Case of Terminological Transfer and Text Translation. In R. Lanselle et al. (Ed.), Translation in an International Perspective. Frankfurt-am-Main/ Berlin/Bern/Bruxelles/New York/Oxford/Wien: Peter Lang.

Formigari, L. (2007). Introduzione alla filosofia delle lingue. Bari: Laterza. 
Fortis, J. M. (sous presse). Bloomfield et l'héritage de la psychologie. Journée d'études La "représentation» entre psychologie et linguistique: aux sources de la sémantique contemporaine. Université de Bourgogne, Dijon. 7/10/2013.

Gardiner, A. H. (1989). Langage et acte de langage. Aux sources de la pragmatique. Traduction et préface par C. Douay. Lille: Presses Universitaires de Lille. (orig. pub. 1932)

Gardiner, Sir A. (1932). The Theory of Speech and Language. Oxford: Oxford University Press.

Hagège, C. (1986). La structure des langues. Paris: PUF «Que sais-je?».

Hœnigswald, R. (1920). Die Grundlagen der Denkpsychologie. Studien und Analysen. Leipzig/ Berlin: B.G. TeubnerVerlag.

Koschmieder, E. (1971[1929]). Zeitbezug und Sprache. Ein Beitrag zur Aspekt und Tempusfrage. Hamburg: Felix Meiner Verlag, Wissenschaftliche Buchgesellschaft.

Koschmieder, E. (1996). Les rapports temporels fondamentaux et leur expression linguistique. Traduction et commentaire par Didier Samain. Villeneuve d'Ascq: Presses du Septentrion. (orig. pub. 1929)

Maturana, H. R. (1987). Ontology of Observing. The Biological Foundations of Self-Consciousness and the Physical Domain of Existence. In E. Gaianiello (Ed.), Physics of cognitive processes. Proceedings of the International Symposium (pp. 324-379). Singapore/ New Jersey/Hong Kong: World Scientific Publishing Co Pte Ltd.

Maturana, H. R., Mpodozis, J., \& Letelier, J. C. (1995). Brain, Language, and the Origin of Human Mental Functions. Biological Research, 28, 15-26.

Nichter, M. (1981). Idioms of Distress: Alternatives in the Expression of Psychosocial Distress. A Case Study from South India. Culture, Medicine and Psychiatry, 5, 379-408.

Prunč, E. (2007). Entwicklungslinien der Translationswissenschaft. Von der Asymetrien der Sprachen zu den Asymetrien der Macht. Berlin: Frank \& Timme GmbH, Verlag für wissenschaftliche Literatur.

Quine, W. v. O. (1960). Word and Object. Cambridge, MA: MIT Press.

Rastier, F. (1995). Communication ou transmission? Césure, 8, 151-198.

Ricœur, P. (2004). Sur la traduction. Paris: Bayard.

Samain, D. (2011). Questions de langue ou histoire de choses. Observations sur la traduction et ses structures élémentaires. In C. Berner \& T. Milliaresi (Éd.), La traduction, philosophie et tradition: interpréter-traduire (pp. 55-78). Villeneuve d'Ascq: Presses universitaires du Septentrion.

Samain, D. (2014, sous presse). La «linguistique» et ses observables. L'exemple des néogrammairiens. In Selected papers from Ichols XII. Amsterdam/Philadelphia: John Benjamins Publishing Company.

Wegener, P. (1991[1885]). Untersuchungen über die Grundfragen des Sprachlebens. Newly edited with an introduction by Clemens Knobloch, by Konrad Koerner. Amsterdam/ Philadelphia: John Benjamins Publishing Company.

Mots-clés: Effet de seuil, histoire des sciences humaines, métalangage, reformulation, terminologie

\section{Übersetzung als entropisches bzw. negentropisches Vorgehen}

\section{Zusammenfassung}

Sich auf seine Erfahrung als Übersetzer und Wissenschaftshistoriker stützend, untersucht der Verfasser die Besonderheiten der geisteswissenschaftlichen Begriffe und deren Übersetzung bzw. Umformulierung. Durch Anwendung von deskriptiven Metabegriffen (Kognitiver Schnitt, Schwelleneffekt) zeigt er, dass die Übersetzung ebenso Informationsgewinn als auch -verlust bringt. 
Ohne die Bedeutung kulturorientierter Analysen in Abrede zu stellen, schlägt er also ein mehr historisches und formelles Vorgehen vor, das vom Gemeinplatz der Äquivalenz zwischen Original und dessen sogenannter Kopie ablässt und die Übertragungmechanismen metasprachlich auffasst. Ausserdem betont die Behandlung die Vieldeutigkeit des Begriffs „Übersetzung“, wie man es am Beispiel des Quines' Begriffs der radikalen Übersetzung veranschaulichen kann.

Schlagworte: Metasprache, Geschichte der Geisteswissenschaften, Schwelleneffekt, Terminologie, Umformulierung.

\section{Entropia e negentropia nella traduzione.}

\section{Riassunto}

L'autore fonda il suo contributo sulla propria esperienza come traduttore e storico, e si concentra sulla specificità dei concetti delle scienze umane e quindi sulla loro traduzione e parafrasi. Usando metaconcetti descrittivi (come troncatura cognitiva e effetto soglia) l'autore mostra come la traduzione implichi sia guadagni sia perdite di informazione. Senza misconoscere il valore dell'analisi culturalista, l'articolo propone un approccio più storico e formale, eliminando $\mathrm{i}$ luoghi comuni legati ai concetti di «originale» e «copia» in favore di una riconsiderazione dei meccanismi di parafrasi come processo metadiscorsivo. Inoltre l'analisi mette in risalto l'ambiguità dell'idea stessa di «traduzione», come illustrato dalla nozione di traduzione radicale di Quine.

Parole chiave: Effetto soglia, metalinguaggio, parafrasi, storia delle scienze umane, terminologia.

\section{Entropy and Negentropy in Translation}

\section{Summary}

The author bases his paper on his experience as a translator and historian and focuses on the specificity of the concepts in moral sciences and hence of their translation or rewording. Using descriptive metaconcepts (such as cognitive truncation and boundary effect), he shows that translation implies both information gains and losses of it.

Though not denying the interest of culturalist analyzes, he thus proposes a more historical and formal approach, getting rid of common places about 'original' and 'copy' in favour of seeing the rewording mechanisms as metadiscursive processes. Besides the analysis emphasizes the ambiguity of the idea of "translation», as it is illustrated by the Quinian notion of radical translation.

Key-words: Boundary effect, history of moral sciences, metalanguage, rewording, terminology. 